\title{
Peroxygenase activity of cytochrome $c$ peroxidase and three apolar distal heme pocket mutants: hydroxylation of 1-methoxynaphthalene
}

\author{
James E Erman*, Heather Kilheeney, Anil K Bidwai, Caitlan E Ayala and Lidia B Vitello
}

\begin{abstract}
Background: The cytochrome P450s are monooxygenases that insert oxygen functionalities into a wide variety of organic substrates with high selectivity. There is interest in developing efficient catalysts based on the "peroxide shunt" pathway in the cytochrome P450s, which uses $\mathrm{H}_{2} \mathrm{O}_{2}$ in place of $\mathrm{O}_{2} / \mathrm{NADPH}$ as the oxygenation agent. We report on our initial studies using cytochrome c peroxidase (CcP) as a platform to develop specific "peroxygenation" catalysts.

Results: The peroxygenase activity of CCP was investigated using 1-methoxynaphthalene as substrate. 1Methoxynaphthalene hydroxylation was monitored using Russig's blue formation at standard reaction conditions of $0.50 \mathrm{mM}$ 1-methoxynaphthalene, $1.00 \mathrm{mM} \mathrm{H}_{2} \mathrm{O}_{2}, \mathrm{pH} 7.0,25^{\circ} \mathrm{C}$. Wild-type $\mathrm{CcP}$ catalyzes the hydroxylation of 1methoxynaphthalene with a turnover number of $0.0044 \pm 0.0001 \mathrm{~min}^{-1}$. Three apolar distal heme pocket mutants of CCP were designed to enhance binding of 1-methoxynaphthalene near the heme, constructed, and tested for hydroxylation activity. The highest activity was observed for CcP(triAla), a triple mutant with Arg48, Trp51, and His52 simultaneously mutated to alanine residues. The turnover number of $\mathrm{CCP}$ (triAla) is $0.150 \pm 0.008 \mathrm{~min}^{-1}, 34$-fold greater than wild-type $\mathrm{CCP}$ and comparable to the naphthalene hydroxylation activity of rat liver microsomal cytochrome P450. While wild-type CcP is very stable to oxidative degradation by excess hydrogen peroxide, CcP (triAla) is inactivated within four cycles of the peroxygenase reaction.

Conclusions: Protein engineering of CCP can increase the rate of peroxygenation of apolar substrates but the initial constructs are more susceptible to oxidative degradation than wild-type enzyme. Further developments will require constructs with increased rates and selectivity while maintaining the stability of wild-type CcP toward oxidative degradation by hydrogen peroxide.
\end{abstract}

Keywords: Cytochrome c peroxidase, Peroxygenase activity, Heme pocket mutants, 1-methoxynaphthalene

\section{Background}

The cytochrome P450s form a large class of heme enzymes that catalyze hydroxylation or epoxidation of organic substrates (S) using molecular oxygen, Equation 1 [1-5]. During the

$$
\begin{gathered}
\mathrm{S}+\mathrm{O}_{2}+\mathrm{NADPH}+\underset{\mathrm{H}}{\mathrm{H}^{+} \rightarrow \mathrm{SO}}+\mathrm{NADP}^{+}+\mathrm{H}_{2} \mathrm{O} \\
\text { P45 }
\end{gathered}
$$

catalytic cycle, molecular oxygen binds to the ferrous

\footnotetext{
* Correspondence: jerman@niu.edu

Department of Chemistry and Biochemistry, Northern Illinois University, DeKalb, IL 60115, USA
}

heme iron. Electron transfer from NADPH generates an enzyme intermediate called Compound I, which retains one of the oxygen atoms from $\mathrm{O}_{2}$ as a ferryl, porphyrinradical species [6]. The oxygen atom from the ferryl heme is transferred to the organic substrate generating the oxygenated product. The rate of cytochrome P450-catalyzed reactions are generally slow with liver microsomal P450s having hydroxylation rates on the order of $1 \mathrm{~min}^{-1}$ while bacterial cytochrome P450s tend to have faster rates $[2,7,8]$.

Cytochrome P450s have been used for synthetic purposes to insert oxygen functionalities with high selectivity into a wide variety of organic substrates $[7,9]$. However, they are not ideal synthetic catalysts. Cytochrome P450 requires an expensive cofactor in NADPH, generally has a 
low turnover rate, and is susceptible to oxidative degradation during the catalytic cycle. One of the first strategies to improve the P450s as synthetic catalysts involved elimination of the requirement for NADPH. Cytochrome P450 can react with $\mathrm{H}_{2} \mathrm{O}_{2}$ to oxygenate substrates in a pathway called the "peroxide shunt" Equation 2 [9]. The peroxide shunt involves $\mathrm{H}_{2} \mathrm{O}_{2}$ reacting

$$
\begin{gathered}
\mathrm{S}+\mathrm{H}_{2} \mathrm{O}_{2} \rightarrow \mathrm{P} \\
\mathrm{P} 450
\end{gathered}
$$

with the Fe(III) heme to generate Compound I. The peroxide shunt pathway is generally slower than the monooxygenase pathway and the enzyme is subject to oxidative degradation by the $\mathrm{H}_{2} \mathrm{O}_{2}$. Protein engineering approaches have been used to enhance the "peroxygenase" activity of the cytochrome P450s [10] as well as to incorporate peroxygenase activity into other heme proteins such as myoglobin and the peroxidases [11,12]. Recently, naturally occurring peroxygenases with high rates of activity have been discovered $[13,14]$.

The peroxidases are a natural platform to explore peroxygenation reactions since the peroxidases have evolved to react efficiently with hydrogen peroxide and many are quite stable to oxidative degradation in the presence of excess hydrogen peroxide. Miller et al. [15] have shown that cytochrome $c$ peroxidase $(\mathrm{CcP})$ and a $\mathrm{CcP}$ mutant, $\mathrm{CcP}(\mathrm{W} 51 \mathrm{~A})$, have low-levels of peroxygenase activity, catalyzing the epoxidation of styrene and styrene derivatives by $\mathrm{H}_{2} \mathrm{O}_{2}$. The turnover numbers for epoxidation of trans- $\beta$-methylstyrene and cis- $\beta$-methylstyrene are about two orders of magnitude slower than the cytochrome P450 monooxygenase reaction.

One factor for the low peroxygenase activity of CcP may be the low affinity for apolar substrates within the heme pocket. Mutating the distal heme pocket to increase the binding of apolar substrates should increase the peroxygenase activity of $\mathrm{CcP}$. In this study we report on the CcP-catalyzed steady-state hydroxylation of 1methoxynaphthalene by $\mathrm{H}_{2} \mathrm{O}_{2}$ using the Russig's blue assay developed by Shoji et al. [12]. Three CcP mutants with apolar distal heme pockets were designed to increase the solubility of typical monooxygenase and peroxygenase substrates within the distal heme pocket of CcP. They were constructed by simultaneously replacing Arg48, Trp51, and His52 with either all alanines, CcP(triAla), all valines, $\mathrm{CcP}$ (triVal), or all leucines, $\mathrm{CcP}($ triLeu). We have previously reported on the reaction of these mutants with hydrogen peroxide and cyanide [16]. As anticipated, the reaction with $\mathrm{H}_{2} \mathrm{O}_{2}$ is substantially reduced but the peroxygenase activity is increased by a factor of 34 .

\section{Methods}

\section{Proteins}

Both authentic yeast cytochrome $c$ peroxidase, $\mathrm{yCcP}$, and a recombinant $\mathrm{CcP}$ with the exact amino acid sequence of $\mathrm{yCcP}, \mathrm{rCcP}$, were used in this study. Isolation and purification of $\mathrm{CcP}$ has been described previously [16,17]. The cloning, expression and purification of $\mathrm{CcP}$ mutants has been described [16,18-21]. The three CcP triple mutants constructed for this study are $\mathrm{CcP}($ triAla $)$ - (R48A/ W51A/H52A), CcP(triVal) - (R48V/W51V/H52V), and CcP(triLeu) - (R48L/W51L/H52L).

\section{Other materials}

Potassium phosphate salts, potassium acetate, and hydrogen peroxide (30\%) were obtained from Fisher Scientific. Hydrogen peroxide solutions were standardized by titration with Ce(IV) as described previously [22]. 1-Methoxynaphthalene was obtained from Aldrich Chemical Company.

\section{Spectroscopic measurements and protein concentration determination}

Spectra of protein solutions were determined using either a Varian/Cary Model 3E or a Hewlett Packard Model 8452A spectrophotometer. Protein concentrations were determined from the absorption spectra using the maximum extinction coefficients in the Soret region at $\mathrm{pH} 6$ : yCcP $98 \mathrm{mM}^{-1} \mathrm{~cm}^{-1}$ at $408 \mathrm{~nm}, \mathrm{rCcP} 101 \mathrm{mM}^{-1} \mathrm{~cm}^{-1}$ at $408 \mathrm{~nm}$, CcP(triAla) $110 \mathrm{mM}^{-1} \mathrm{~cm}^{-1}$ at $406 \mathrm{~nm}, \mathrm{CcP}$ (triVal) $76 \mathrm{mM}^{-1} \mathrm{~cm}^{-1}$ at $406 \mathrm{~nm}$, and $\mathrm{CcP}$ (triLeu) $93 \mathrm{mM}^{-1} \mathrm{~cm}^{-1}$ at $400 \mathrm{~nm}[16,17]$.

\section{Steady-state kinetic measurements of 1-methoxynaphthalene hydroxylation}

1-Methoxynaphthalene hydroxylation was monitored by following formation of Russig's blue at $610 \mathrm{~nm}$ [12]. A reaction mechanism for Russig's blue formation is shown in Figure 1. The initial change in absorbance is converted to an initial velocity, $v_{0}$, using Equation 3. The change

$$
v_{0}=2(\mathrm{dA} / \mathrm{d} t) / \Delta \varepsilon
$$

in extinction coefficient, $\Delta \varepsilon$, for Russig's blue formation in aqueous solution is $1.45 \times 10^{4} \mathrm{M}^{-1} \mathrm{~cm}^{-1}$ at $610 \mathrm{~nm}$ ( in the Additional file 1: Figure SA.1). The factor of 2 in Equation 3 is required since two molecules of 1methoxynaphthalene must be hydroxylated to produce one molecule of the product, Russig's blue (Figure 1). Standard assay conditions were $0.50 \mathrm{mM}$ 1methoxynaphthalene, $1.0 \mathrm{mM}$ hydrogen peroxide, enzyme as required (generally in the $\mu \mathrm{M}$ concentration range), $\mathrm{pH} 7.0,0.10 \mathrm{M}$ ionic strength potassium phosphate buffer, $25^{\circ} \mathrm{C}$. The reaction was initiated by addition of hydrogen peroxide. 


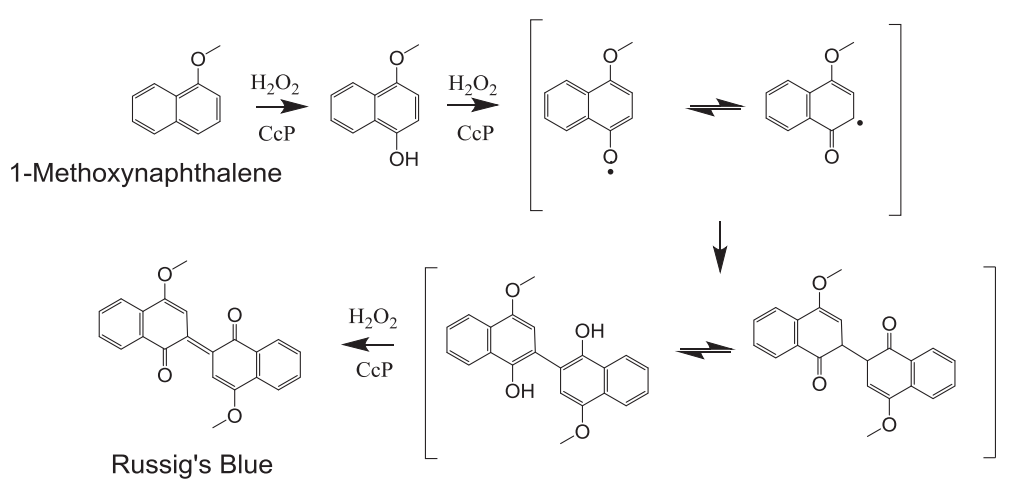

Figure 1 Proposed reaction sequence for conversion of 1-methoxynaphthalene to Russig's blue. Adapted from reference [12].

\section{Results}

Steady-state hydroxylation of 1-methoxynaphthalene by $\mathrm{CCP}$ and the apolar distal heme pocket mutants of CcP We have utilized the Russig's blue assay developed by Shoji and coworkers [12] to assess the 1methoxynaphthalene hydroxylation activity of $\mathrm{CcP}$ and the three $\mathrm{CcP}$ mutants with apolar distal heme pockets, $\mathrm{CcP}$ (triAla), $\mathrm{CcP}($ triLeu), and $\mathrm{CcP}($ triVal). Figure 2 shows the $\mathrm{CcP}$ (triAla) catalyzed oxidation of 1 methoxynaphthalene by hydrogen peroxide at $\mathrm{pH}$ 7.0. There is a large increase in the absorbance between 500 and $800 \mathrm{~nm}$ during the reaction that is characteristic of Russig's blue formation resulting from the hydroxylation of 1-methoxynaphthalene, Figure 1 [12]. The inset of Figure 2 shows the increase in the absorbance at
$610 \mathrm{~nm}$ as a function of time from which the initial velocity can be determined, Equation 3 . The initial velocity increases linearly with increasing $\mathrm{CcP}($ triAla) concentration, Figure 3, from which a turnover number of $0.150 \pm 0.008 \mathrm{~min}^{-1}$ can be determined, Table 1 .

Spectral scans of the $\mathrm{CcP}$ (triLeu), CcP(triVal), and rCcP catalyzed Russig's blue formation are similar to those for $\mathrm{CcP}$ (triAla) and are shown in the Additional file 1 associated with this manuscript. Initial velocities as a function of the $\mathrm{CcP}($ triLeu) and $\mathrm{rCcP}$ concentration are shown in Figure 3, along with the $\mathrm{CcP}$ (triAla) data. The initial velocities increases linearly with increasing $\mathrm{CcP}($ triLeu) and $\mathrm{rCcP}$ concentrations. Turnover numbers are included in Table 1. The initial velocity for the $\mathrm{CcP}($ triVal)-catalyzed oxidation of 1-methoxynaphthalene is independent of the CcP

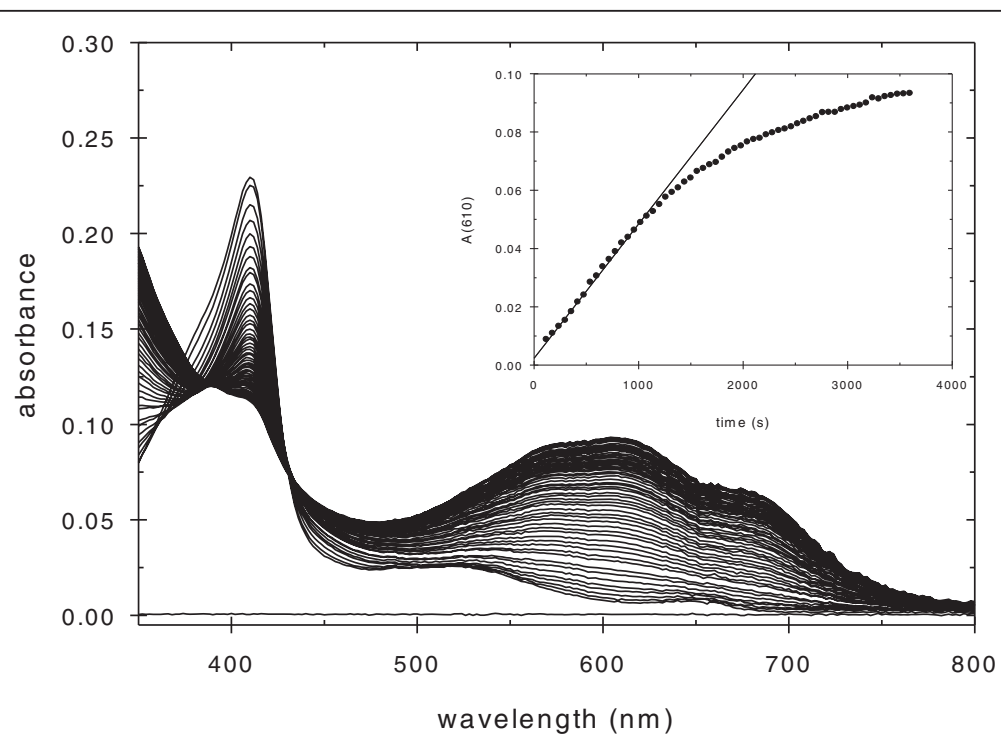

Figure 2 CcP(triAla)-catalyzed oxidation of 1-methoxynaphthalene by hydrogen peroxide. Spectra were collected every minute for one hour after addition of hydrogen peroxide. The large increase in absorbance between 450 and $800 \mathrm{~nm}$ is due to Russig's blue formation. Inset - Increase in the absorbance at $610 \mathrm{~nm}$ as a function of time after hydrogen peroxide addition. Experimental Conditions: $[\mathrm{CcP}($ triAla $)]=2.0 \mu \mathrm{M},[1$-methoxynaphthalene $]=0.50 \mathrm{mM},\left[\mathrm{H}_{2} \mathrm{O}_{2}\right]=1.00 \mathrm{mM}, \mathrm{pH} 7.0,0.100 \mathrm{M}$ ionic strength potassium phosphate buffer, $25^{\circ} \mathrm{C}$. 


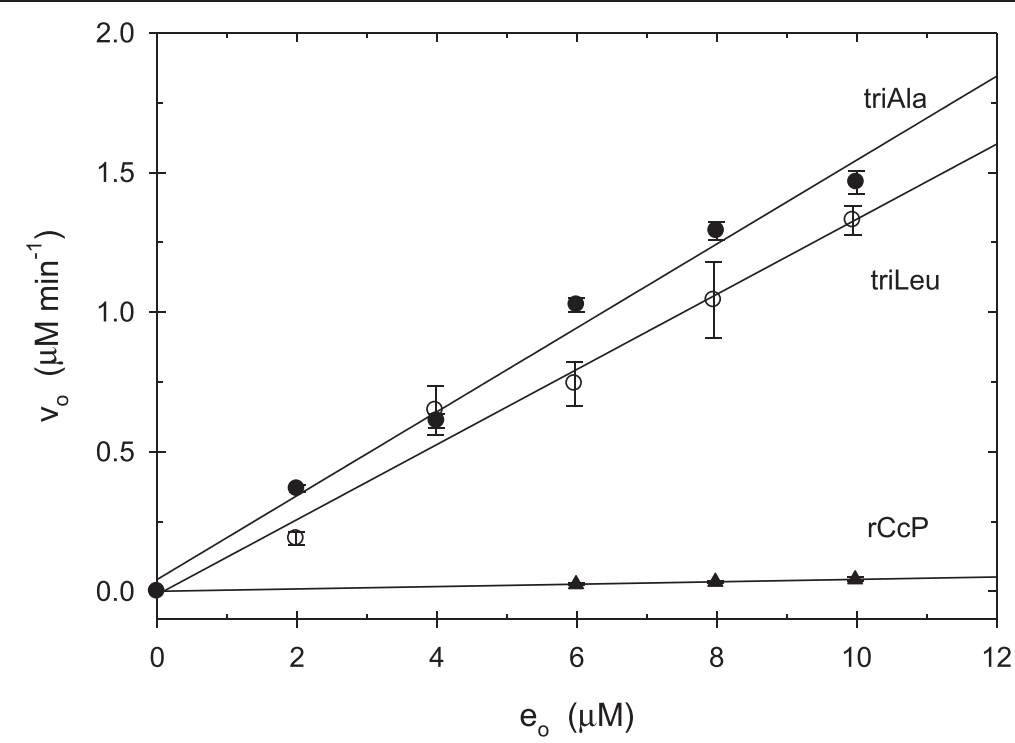

Figure 3 Plots of initial velocity, $v_{0}$, for 1-methoxynaphthalene hydroxylation as a function of initial enzyme concentration, $e_{0}$. CcP (triAla) - solid circles, CcP(triLeu) - open circles, rCcP - solid triangles. Experimental Conditions: [1-methoxynaphthalene] $=0.50 \mathrm{mM}$, $\left[\mathrm{H}_{2} \mathrm{O}_{2}\right]=1.00 \mathrm{mM}, \mathrm{pH} 7.0,0.100 \mathrm{M}$ ionic strength potassium phosphate buffer, $25^{\circ} \mathrm{C}$.

(triVal) concentration between 5 and $10 \mu \mathrm{M}$ and only a minimum value of $0.137 \mathrm{~min}^{-1}$ can be established for the $\mathrm{CcP}($ triVal) turnover rate.

The rate of 1-methoxyhaphthalene hydroxylation catalyzed by $\mathrm{CcP}$ (triAla) was studied as functions of the 1-methoxynaphthalene and hydrogen peroxide concentrations. The initial velocity increases linearly with 1-methoxynaphthalene concentration and with the hydrogen peroxide concentrations (Additional file 1). Apparent second-order rate constants for the interaction of 1-methoxynaphthalene with $\mathrm{CcP}$ (triAla) Compound I and of hydrogen peroxide with $\mathrm{CcP}$ (triAla) are $1.5 \pm 0.2 \mathrm{M}^{-1} \mathrm{~s}^{-1}$ and $1.1 \pm 0.1 \mathrm{M}^{-1} \mathrm{~s}^{-1}$, respectively.

At the high concentrations of hydrogen peroxide used in the Russig's blue assay, significant bleaching of the Soret absorption band occurs during the reaction, Figure 2, indicating heme destruction throughout the assay. This diminishes the catalytic effectiveness of $\mathrm{CcP}$ and its mutants. In a study of the hydroxylation of 1-metholxynaphthalene catalyzed by $\mathrm{CcP}$ (triAla), the formation of Russig's blue was followed until cessation of the reaction. Using $10 \mu \mathrm{M}$ CcP(triAla), $19.5 \mu \mathrm{M}$ Russig's blue was formed, corresponding to the hydroxylation of $39 \mu \mathrm{M} 1$ methoxynaphthalene.

\section{Screening of heme pocket mutants of CcP for} 1-methoxynaphtalene hydroxylation activity

We screened all of the $\mathrm{CcP}$ heme pocket mutants that were available in our laboratory for 1-methoxynaphthalene activity to determine if any had unusually high peroxygenase activity or higher stability toward heme degradation during the peroxygenase reaction. The results are shown in Figure 4. Turnover numbers for the CcP mutants not specifically designed to bind apolar substrates ranged from $0.0038 \pm 0.0007 \mathrm{~min}^{-1}$ for $\mathrm{CcP}(\mathrm{H} 52 \mathrm{Q})$ to $0.092 \pm 0.004$ $\min ^{-1}$ for $\mathrm{CcP}(\mathrm{D} 235 \mathrm{~K})$. Two $\mathrm{CcP}$ mutants designed to mimic the heme axial ligation of cytochrome $\mathrm{P} 450, \mathrm{CcP}$ $(\mathrm{H} 175 \mathrm{C})$ and $\mathrm{CcP}(\mathrm{H} 175 \mathrm{C} / \mathrm{D} 235 \mathrm{~L})$, have hydroxylation rates of $0.036 \pm 0.001$ and $0.026 \pm 0.001 \mathrm{~min}^{-1}$, respectively. Turnover numbers $\left(v_{0} / e_{0}\right)$ for all $\mathrm{CcP}$ mutants determined in this study are collected in the Additional file 1: Table SA.1. Heme degradation for all mutants is shown in Additional file 1: Figure SA.8. $\mathrm{CcP}(\mathrm{H} 52 \mathrm{~N})$ is most resistant to heme degradation during the peroxygenase reaction while $\mathrm{CcP}(\mathrm{W} 51 \mathrm{H})$ is most susceptible.

\section{Discussion}

Relatively few studies of naphthalene hydroxylation by the cytochrome P450s have been reported. Rat liver

Table 1 Peroxygenase activity of ccp and ccp mutants

\begin{tabular}{ccccc}
\hline Enzyme & Substrate & Substrate concentration $(\mathbf{m M})$ & pH & TN $\left(\mathbf{m i n}^{-1}\right)$ \\
\hline rCcP & 1-methoxynaphthalene & 0.50 & 7.0 & $0.0044 \pm 0.0001$ \\
CcP(triAla) & 1-methoxynaphthalene & 0.50 & 7.0 & $0.150 \pm 0.008$ \\
CcP(trival) & 1-methoxynaphthalene & 0.50 & 7.0 & $>0.137$ \\
CcP(triLeu) & 1-methoxynaphthalene & 0.50 & 7.0 & $0.134 \pm 0.014$ \\
\hline
\end{tabular}




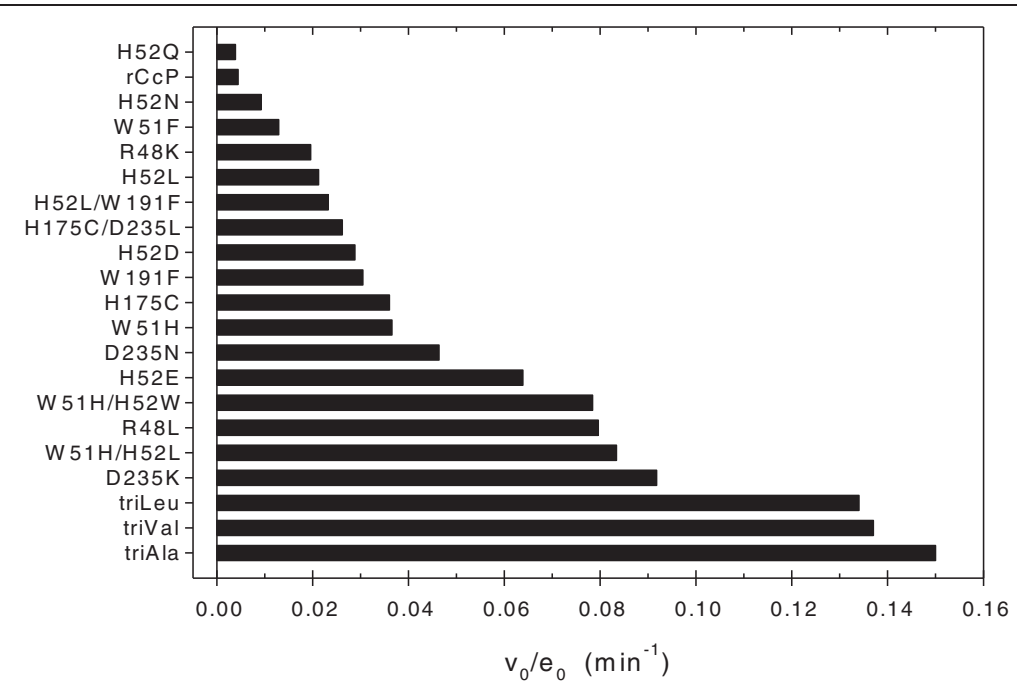

Figure 4 Turnover rates, $v_{0} / e_{0}$, for 1 -methoxynaphthalene hydroxylation by hydrogen peroxide catalyzed by $\mathrm{rCcP}$ and $17 \mathrm{CcP}$ mutants. Experimental Conditions: [1-methoxynaphthalene] $=0.50 \mathrm{mM},\left[\mathrm{H}_{2} \mathrm{O}_{2}\right]=1.00 \mathrm{mM}, \mathrm{pH} 7.0,0.100 \mathrm{M}$ ionic strength potassium phosphate buffer, $25^{\circ} \mathrm{C}$.

microsomes hydroxylate naphthalene with a monooxygenation turnover rate of $0.32 \mathrm{~min}^{-1}[23]$ and cytochrome $\mathrm{P} 450_{\text {cam }}$ hydroxylates naphthalene with a rate of $0.7 \mathrm{~min}^{-1}[24]$. Protein engineering of cytochrome $\mathrm{P} 450_{\text {cam }}$ produced a Y96F mutant, which increases the rate of naphthalene hydroxylation to $100 \mathrm{~min}^{-1}$ [24], the fastest reported for a cytochrome P450. Joo et al. have been using laboratory evolution techniques to enhance the peroxygenase activity of cytochrome P450 cam $_{\text {[10]. Using a }}$ fluorescence technique to monitor naphthalene hydroxylation, Joo and colleagues screened approximately 200,000 random mutants of cytochrome $\mathrm{P} 450_{\text {cam }}$ and were able to select a mutant that had 11-fold higher peroxygenase activity than did the wild-type enzyme although absolute rates were not reported.

Cytochrome $\mathrm{P} 450_{\mathrm{BS} \beta}$ is a naturally-occurring peroxygenase, which hydroxylates fatty acids predominantly at the $\beta$ position [14]. It has no monooxygenase activity and does not react with hydrogen peroxide in the absence of the fatty acid substrate. Myristic acid is the preferred substrate and the turnover rate is about $300 \mathrm{~min}^{-1}$. In the presence of a short-chain fatty acid, cytochrome $\mathrm{P} 450_{\mathrm{BS} \beta}$ will catalyze the peroxygenation of a variety of nonnatural substrates such as styrene, ethylbenzene, and 1-methoxynaphthalene.

Shoji et al. [12] investigated the rates of peroxygenation of 1-methoxynaphthalene by three heme proteins, cytochrome $\mathrm{P} 450_{\mathrm{BS} \beta}$, HRP, and metmyoglobin. HRP had no detectable activity, while sperm whale metmyoglobin has a turnover number of about $0.03 \mathrm{~min}^{-1}$. The H64A mutation of metmyoglobin increases the rate of Russig's blue formation almost 800 -fold to $23 \mathrm{~min}^{-1}$. Cytochrome $\mathrm{P} 450_{\mathrm{BS} \beta}$ had a turnover number of $112 \mathrm{~min}^{-1}$ for 1-methoxynaphthalene hydroxylation.
In this study, we show that $\mathrm{CcP}$ has detectable hydroxylation activity with a turnover number of $0.0044 \mathrm{~min}^{-1}$, faster than that of HRP but slower than that of metMb and cytochrome $\mathrm{P} 450_{\mathrm{BS} \beta}$. The three $\mathrm{CcP}$ mutants designed to bind non-polar substrates within the heme pocket, $\mathrm{CcP}$ (triAla), $\mathrm{CcP}$ (triVal), and $\mathrm{CcP}($ triLeu), are about 30 -fold more active than $\mathrm{rCcP}$, however, none approached the activities of metMb(H64A) or cytochrome $\mathrm{P} 450_{\mathrm{BS} \beta}$. Two $\mathrm{CcP}$ mutants, $\mathrm{H} 175 \mathrm{C}$ and H175C/D235L, designed to simulate the heme ligation in cytochrome P450 have peroxygenase activities that are intermediate between those of $\mathrm{rCcP}$ and $\mathrm{CcP}$ (triAla).

One of the anticipated strengths of $\mathrm{CcP}$ as a platform to develop specific peroxygenase catalysts is the stability of the initial oxidized intermediate in the $\mathrm{CcP} /$ hydrogen peroxide reaction, CcP Compound I, and the stability with respect to oxidative degradation by excess hydrogen peroxide $[25,26]$. The half-life of $\mathrm{CcP}$ compound I is about 6 hours at $\mathrm{pH} 6$ [25] and $\mathrm{CcP}$ can react with up to 10 equivalents of hydrogen peroxide in the absence of oxidizable substrates before significant reduction in catalytic activity occurs [26]. However, in the Russig's blue assay, we saw rapid bleaching of the heme, Figure 2, and complete inactivation of $\mathrm{CcP}$ (triAla) within four catalytic cycles. In our screening process, we found that $\mathrm{CcP}$ (triAla) is one of the most susceptible mutants to heme degradation with a loss of $73 \%$ of the Soret absorbance during the Russig's blue assay, in the Additional file 1: Figure SA.8. Interestingly, there is about a 10 -fold variation in heme degradation during the Russig's blue assay for the twenty CcP mutants screened in this study, Figure A8. $\mathrm{CcP}(\mathrm{H} 52 \mathrm{~N})$ is the most stable of the screened mutants with only $8 \%$ heme degradation while $\mathrm{CcP}(\mathrm{W} 51 \mathrm{H})$ is the most susceptible mutant with $74 \%$ heme degradation. The 
degradation does not correlate with either the rate of compound I formation or with the rate of 1methoxynaphthalene hydroxylation. It may be due to secondary oxidation of the hydroxylated product within the distal heme pocket, Figure 1, forming substrate-based radicals which, in turn, react with the heme causing the observed degradation.

\section{Conclusions}

The 1-methoxynaphthalene hydroxylation activity of wildtype $\mathrm{CcP}$ can be increased 34-fold by making the distal heme pocket apolar. The 1-methoxynaphthalene hydroxylation rate of $0.150 \pm 0.008 \mathrm{~min}^{-1}$ for $\mathrm{CcP}$ (triAla) is only a factor of two smaller than the naphthalene hydroxylation activity of rat liver microsomal cytochrome P450 but is still three orders of magnitude slower than that of the best naphthalene hydroxylation catalysts yet reported, the peroxygenation of 1-methoxynaphthalene by cytochrome $\mathrm{P} 450_{\mathrm{BS} \beta}$ with a turnover number of $112 \mathrm{~min}^{-1}$ [12] and the monooxygenase activity of cytochrome $\mathrm{P}_{450_{\text {CAM }}(\mathrm{Y} 96 \mathrm{~F})}$ with a turnover number of $100 \mathrm{~min}^{-1}$ [24]. The increased rate of 1-methoxynaphthalene hydroxylation comes at the expense of decreased stability of CcP toward oxidative degradation in the presence of excess hydrogen peroxide. Further development of $\mathrm{CcP}$ mutants as peroxygenation catalysts will have to maintain the stability of the enzyme while increasing the rate of substrate turnover.

\section{Availability of supporting data}

All supporting data are included in the additional file included with this article.

\section{Additional file}

Additional file 1: The additional file provided with this paper contains 8 figures and 1 table. Figure SA.1. Spectrum of Russig's blue in organic and aqueous solution. Figure SA.2, Figure SA.2, and Figure SA.3. Spectral scans CCP(triLeu)-, CCP(triVal)-, and rCcP-catalyzed formation of Russig's blue, respectively. Figure SA.5. Dependence of the initial velocity for CCP(triAla)-catalyzed formation of Russig's blue on the concentration of 1-methoxynaphthalene. Figure SA.6. Dependence of the initial velocity for $\mathrm{CCP}($ triAla)-catalyzed formation of Russig's blue on the concentration of hydrogen peroxide. Figure SA.7. Histogram of the peroxygenase activity of rCcP, 20 CcP mutants, and selected other heme proteins. Figure SA.8. Percent heme degradation during the peroxygenase reaction for the heme proteins included in Figure SA.7 Table SA.1. Peroxygenase activity of $\mathrm{rCCP}, 20 \mathrm{CcP}$ mutants, and selected other heme proteins.

\section{Abbreviations}

CCP: Generic abbreviation for cytochrome c peroxidase whatever the source; yCcP: Authentic yeast cytochrome $c$ peroxidase isolated from S. cervisiae; rCcP: Recombinant cytochrome $c$ peroxidase expressed in E. coli, which has an identical amino acid sequence to that of $\mathrm{yCCP} ; \mathrm{CcP}($ triAla): Triple mutant of $\mathrm{rCCP}$ with R48A/W51A/H52A; CCP(triLeu): Triple mutant of rCCP with R48L/W51L/H52V; CcP(triVal): Triple mutant of rCcP with R48V/W51V/H52V.

\section{Competing interests}

All authors declare that they have no competing interests.

\section{Authors' contributions}

JEE proposed and designed the experiments, carried out some of the Russig's blue assays, and wrote the initial draft of the manuscript. LBV was responsible for constructing the triple mutants. AKB performed the initial characterization of the triple mutants. CEA helped develop the initial testing protocol including determining the spectra of Russig's blue in aqueous solution. HK helped characterize the triple mutants and was involved in the Russig's blue assays. All authors were involved in interpretation of the data, editing the manuscript, and all have read and approved the final manuscript.

\section{Acknowledgements}

This work was supported in part by the National Institutes of Health through grant R15 GM59740. We thank Professor Yi Lu from the University of Illinois, Champaign-Urbana for providing samples of $\mathrm{CCP}(\mathrm{H} 175 \mathrm{C})$ and $\mathrm{CCP}(\mathrm{H} 175 \mathrm{C} / \mathrm{D} 235 \mathrm{~L}$.

Received: 15 April 2013 Accepted: 25 July 2013

Published: 30 July 2013

\section{References}

1. de Montellano PR O (Ed): Cytochrome P-450. New York: Plenum Press; 1986.

2. de Montellano PR O (Ed): Cytochrome P-450: structure, mechanism, and biochemistry. 2 editionth edition. New York: Plenum Press; 1995.

3. de Montellano PR O (Ed): Cytochrome P-450: structure, mechanism, and biochemistry. 3 editionth edition. New York: Plenum Press; 2005.

4. Guengerich FP: Reaction and significance of cytochrome P-450 enzymes. J Biol Chem 1991, 266:10019-10022.

5. Sono M, Roach MP, Coulter ED, Dawson JH: Heme-containing oxygenases. Chem Rev 1996, 96:2841-2887.

6. Rittle J, Green MT: Cytochrome P450 compound I: capture, characterization, and C-H bond activation kinetics. Science 2010, 330:933-937.

7. Martinez CA, Stewart JD: Cytochrome P450's: potential catalysts for asymmetric olefin epoxidations. Curr Org Chem 2000, 4:263-282.

8. Ma Q, Lu AYH: The challenges of dealing with promiscuous drug-metabolizing enzymes, receptors, and transporters. Curr Drug Metab 2008, 9:374-383.

9. van Rantwijk F, Sheldon RA: Selective oxygen transfer catalyzed by heme peroxidases: synthetic and mechanistic aspects. Curr Opin Biotech 2000, 11:554-564.

10. Joo H, Lin Z, Arnold FH: Laboratory evolution of peroxide-mediated cytochrome P450 hydroxylation. Nature 1999, 399:670-673.

11. Watanabe Y: Construction of heme enzymes: four approaches. Curr Opion Chem Biol 2002, 6:208-216.

12. Shoji O, Wiese C, Fujishiro T, Shirataki C, Wünsch B, Watanabe Y: Aromatic $\mathrm{C}-\mathrm{H}$ bond hydroxylation by $\mathrm{P} 450$ peroxygenases: a facile colorimetric assay for monooxygenation activities of enzymes based on Russig's blue formation. J Biol Inorg Chem 2010, 15:1109-1115.

13. Matsunaga I, Yamada M, Kusunose E, Miki T, Ichihara K: Further characterization of hydrogen peroxide-dependent fatty acid a-hydroxylase from Sphingomonas paucimobilis. J Biochem 1998, 124:105-110.

14. Matsunaga I, Ueda A, Fujiwara N, Sumimoto T, Ichihara K: Characterization of the $y b d T$ gene product of Bacillus subtilis novel fatty acid $\beta$-hydroxylating cytochrome P450. Lipids 1999, 34:841-846.

15. Miller VP, DePillis GD, Ferrer JC, Mauk AG, Ortiz de Montellano R: Monooxygenase activity of cytochrome c peroxidase. J Biol Chem 1992, 267:8936-8942.

16. Bidwai AK, Meyen C, Kilheeney H, Wroblewski D, Vitello LB, Erman JE: Apolar distal pocket mutants of yeast cytochrome $c$ peroxidase: hydrogen peroxide reactivity and cyanide binding of the triAla, triVal, and triLeu variants. Biochim Biophys Acta 1834, 2013:137-148.

17. Vitello $L B$, Huang $M$, Erman JE: $p H$-dependent spectral and kinetic properties of cytochrome $c$ peroxidase: comparison of freshly isolated and stored enzyme. Biochemistry 1990, 29:4283-4288.

18. Savenkova MI, Satterlee JD, Erman JE, Siems WF, Helms GL: Expression, purification, characterization, and NMR studies of highly deuterated recombinant cytochrome $c$ peroxidase. Biochemistry 2001, 40:12123-12131.

19. Takio K, Titani K, Ericsson LH, Yonetani T: Primary structure of yeast cytochrome $c$ peroxidase II. The complete amino acid sequence. Arch. Biochem Biophys 1980, 203:615-6299.

20. Teske JG, Savenkova MI, Mauro JM, Erman JE, Satterlee JD: Yeast cytochrome $c$ peroxidase expression in Escherichia coli and rapid 
isolation of various highly purified holoenzymes. Protein Expression Purif 2000, 19:139-147.

21. Fishel LA, Villafranca JE, Mauro JM, Kraut J: Yeast cytochrome $c$ peroxidase: mutagenesis and expression in Escherichia coli show tryptophan-51 is not the radical site in compound I. Biochemistry 1987, 26:351-360.

22. Kolthoff IM, Belcher R: Hydrogen peroxide. In Volumetric analysis, Volume 3. New York: Interscience; 1957:75-76.

23. Booth J, Boyland E: Metabolism of polycyclic compounds. 13. Enzymic hydroxylation of naphthalene by rat-liver microsomes. Biochem J 1958, 70:681-688.

24. England PA, Harford-Cross CF, Stevenson J-A, Rouch DA, Wong L-L: The oxidation of naphthalene and pyrene by cytochrome $P 450_{\text {cam }}$. FEBS Lett 1998, 424:271-274.

25. Erman JE, Yonetani T: A kinetic study of the endogenous reduction of the oxidized sites in the primary cytochrome $c$ peroxidase-hydrogen peroxide compound. Biochem Biophys Acta 1975, 393:350-357.

26. Erman JE, Yonetani $\mathrm{T}$ : The oxidation of cytochrome $c$ peroxidase by hydrogen peroxide. Characterization of products. Biochim Biophys Acta 1975, 393:343-349.

doi:10.1186/1471-2091-14-19

Cite this article as: Erman et al:: Peroxygenase activity of cytochrome $c$ peroxidase and three apolar distal heme pocket mutants: hydroxylation of 1-methoxynaphthalene. BMC Biochemistry 2013 14:19.

\section{Submit your next manuscript to BioMed Central and take full advantage of:}

- Convenient online submission

- Thorough peer review

- No space constraints or color figure charges

- Immediate publication on acceptance

- Inclusion in PubMed, CAS, Scopus and Google Scholar

- Research which is freely available for redistribution 